\title{
Andrade, Susan E. The Nation Writ Small: African Fictions and Feminisms, 1958-1988
}

Durham-London, Duke University Press, 2011

\section{Augustine H. Asaah}

\section{OpenEdition}

\section{Journals}

Édition électronique

URL : http://journals.openedition.org/etudesafricaines/18154

DOI : 10.4000/etudesafricaines. 18154

ISSN : $1777-5353$

\section{Éditeur}

Éditions de l'EHESS

Édition imprimée

Date de publication : 6 juillet 2015

ISSN : 0008-0055

\section{Référence électronique}

Augustine H. Asaah, «Andrade, Susan E. The Nation Writ Small: African Fictions and Feminisms,

1958-1988 », Cahiers d'études africaines [En ligne], 218 / 2015, mis en ligne le 01 janvier 2015, consulté le 24 septembre 2020. URL : http://journals.openedition.org/etudesafricaines/18154 ; DOI : https://doi.org/

10.4000/etudesafricaines. 18154

Ce document a été généré automatiquement le 24 septembre 2020

(c) Cahiers d'Études africaines 


\title{
Andrade, Susan E. The Nation Writ Small: African Fictions and Feminisms,
} 1958-1988

Durham-London, Duke University Press, 2011

\author{
Augustine H. Asaah
}

\section{ANDRADE, Susan E. - The Nation Writ Small : African Fictions and Feminisms, 1958-1988. Durham-London, Duke University Press, 2011,259 p., bibl, ill.}

1 Nourri fondamentalement de réflexions féministes, The Nation Writ Small de Susan Andrade, spécialiste de littérature exerçant aux États-Unis, se focalise sur la place de la sphère familiale dans les récits nationalistes africains et vient approfondir la critique féministe africaine enrichie par des universitaires comme Juliana Makuchi NfahAbbeny ${ }^{1}$, Pierrette Herzberger-Fofana ${ }^{2}$, Kenneth Harrow ${ }^{3}$ et Ayo O.Coly ${ }^{4}$. Sur sa quadripartition en chapitres viennent se greffer une introduction plutôt circonstanciée de quarante-trois pages, une conclusion, une bibliographie, une chronologie sélective de récits, un index et une photo unique. Si les œuvres analysées, comme le laisse interpréter le sous-titre, s'inscrivent dans la période 1958-1988, la chronologie de textes, elle, réunit quarante-six romans africains, majoritairement en langues européennes, sortis entre 1930 et 2006. Pour encore élargir le champ de son analyse, Andrade fait des références aux œuvres comme Jagua Nana de Cyprian Ekwensi et Bones de Chenjerai Hove, tout en s'appuyant sur des ouvrages comme Les damnés de la terre de Frantz Fanon et Imagined Communities de Benedict Anderson.

2 Le corpus de onze textes se compose d'un film et de dix romans créés par trois hommes et six femmes, originaires de cinq pays africains : Algérie, Nigeria, Sénégal, Somalie et Zimbabwé. De ces récits narratifs, cinq sont d'expression anglaise alors que six sont en langue française. Si le corpus réunit dans un assemblage panafricain des œuvres venant 
de diverses aires géographiques et linguistiques, l'auteure se tait pourtant sur les motivations de ses choix.

D'apparence détaillée, l'introduction permet à Andrade d'insérer son analyse dans un contexte sociohistorique et littéraire. Ainsi le lecteur est-il amené à suivre l'évolution de la littérature africaine europhone et à noter aussi bien l'invisibilité des femmes dans la littérature africaine dite nationaliste (œuvres et critique littéraire) rédigée par des hommes que la riposte féminine à cette exclusion. Tout en signalant la continuité entre ces œuvres, Andrade s'attache également à en souligner les points divergents permettant d'évaluer les mutations sociales qui les façonnent.

4 Se voit de plus dans l'introduction un appareillage conceptuel solide. Comme le point de départ de son étude est l'association intime entre le domaine domestique et l'arène politique, Andrade recourt aux thèses marxistes et féministes pour renforcer la présence de l'intime et de l'espace familial dans la chose politique. Le travail domestique, affirme-t-elle, participe d'un travail collectif et sa prise en charge par les femmes libère les sujets masculins pour s'impliquer davantage dans des affaires publiques. D'autre part, comme Une si longue lettre de Mariama Bâ, Nervous Condition de Tsitsi Dangaremgba et Purple Hisbiscus de Chimananda Adichie l'ont amplement illustré, la dictature domestique misogyne est à l'image d'un patriarcat national tyrannique ou d'une politique inopérante ambiante dominée par les hommes. Dans ces conditions, si le sujet lisant aborde la littérature africaine féminine ou féministe en reconnaissant les dimensions allégoriques inéluctables de ces récits, il sera mieux placé, selon elle, pour constater les accents palpables et symboliques des représentations fictionnelles de l'espace familial dans un récit féminin, même le plus apolitique, et par là même, leur apport au discours nationaliste. À cet égard, elle trouve utiles les réflexions formulées par les critiques marxisants, Frederick Jameson et Gayatri C. Spivak. Dans un esprit marxiste et féministe, cette dernière estime que, dans la mesure où le tissu du secteur prétendument politique se fabrique toujours d'éléments privés, l'intime acquiert d'office un potentiel public et national. Andrade s'appuie en particulier sur la notion d'« inconscient politique » et sur l'article polémique, « Third World Literature in an Era of Multinational Capitalism », de Jameson pour qui l'imbrication privé/public s'avère une constante obsessionnelle dans la littérature tiers-mondiste. Ces jalons conceptuels liminaires permettent dès lors à l'auteure d'orienter les notions-clés d'allégorie, de représentation et de généalogie vers la justification du titre de son ouvrage : la famille est le microcosme de la nation dans les œuvres africaines et tout ce qui s'y rapporte mariage, foyer, amour, libido, femme, enfant, tâches ménagères, gestion, etc. s'intègre directement ou indirectement dans la sphère nationale.

C'est sur la base de ces réflexions que l'auteure développe ses arguments dans le corps de l'ouvrage. Dans le premier chapitre, l'absence de femmes igbo de la guerre anticoloniale de 1929 dans l'historiographie africaine et dans les récits nationalistes qui s'ajoute au refus de certains critiques littéraires, africains comme africanistes, de considérer le genre comme catégorie analytique essentielle autorise Andrade à évaluer l'élan subversif féminin dans deux œuvres africaines à savoir, The Joys of Motherhood de Buchi Emecheta et Efuru de Flora Nwapa, premier roman par une Nigériane. À juste titre, l'auteure voit la prise de parole historique par des femmes et la visibilité fictionnelle des femmes comme actes politiques. Autant la révolte féminine contre l'impérialisme britannique se veut explicite chez Emecheta, autant elle s'avère limitée dans le roman de Nwapa. D'autre part, l'exclusion du militantisme anti-patriarcal et 
anticolonial de la part des femmes dans Things Fall Apart de Chinua Achebe, que démentent à la fois l'histoire et la culture igbo, conforte chez cet écrivain, autrement vénéré, la volonté de neutraliser, pour ne pas dire masculiniser, les déesses et rituels féminins igbo dans cette œuvre considérée comme l'un des récits fondateurs du nationalisme africain. Les erreurs d'omission et d'action chez le patriarche des lettres africaines en dit long sur l'intérêt déficitaire que le discours nationaliste africain porte à la famille, à la femme et au genre dans sa configuration des communautés nationales. Sous ce rapport, The Joys of Motherhood de Buchi Emecheta et Efuru déconstruisent les assises patriarcales du métarécit nationaliste. Reformulation du titre antiphrastique du chef d'œuvre d'Emecheta The Joys of Motherhood, l'intitulé du premier chapitre de l'essai d'Andrade The Joys of Daughterhood laisse entendre la filiation qui unit l'hypotexte à son hypertexte.

6 Titré "The Loved and the Left ", le deuxième chapitre explore les liens étroits entre la libido, l'asymétrie sexuelle et sociale, la dégénérescence des syndicalistes/ fonctionnaires naguère progressistes et l'échec des indépendances nationales tels que dépeints dans des échantillons de littérature africaine gauchisante et féministe. Les textes primaires analysés sont Xala (film et roman) d'Ousmane Sembène, La grève des bàttus ou Les déchets humains de Aminata Sow Fall et Une si longue lettre de Mariama Bâ, tous ayant pour cadre un Sénégal qui a une longue histoire d'affaires, parfois ambigües, avec le socialisme depuis le militant Lamine Senghor (auteur de La violation d'un pays) ${ }^{5}$ jusqu'à Léopold Sédar Senghor sans oublier Mamadou Dia et Abdou Diouf. Autant Andrade dans le premier chapitre jette son dévolu sur des romans d'auteurs nigérians, autant elle priorise des œuvres sénégalaises dans le deuxième chapitre. Si mue par une volonté généalogique et intertextuelle, l'auteure rattache La grève des bàttus et Une si longue lettre à Xala et ce, de manière convaincante, elle démontre parallèlement les rapports thématiques et discursifs entre d'une part, les trois romans et le film et, d'autre part, les quatre œuvres et l'essai de Frantz Fanon, Les damnés de la terre, considéré par beaucoup comme l'Ur-texte de la pensée postcoloniale. Dans le chapitre "Les mésaventures de la conscience nationale », Fanon jette une lumière crue sur la fausse conscience de l'élite africaine, les limitations de la bourgeoisie naissante nationale et la cupidité des dirigeants néocolonialistes portés au pouvoir au lendemain des indépendances, éclairage qui permet de mieux apprécier la représentation saisissante des systèmes dysfonctionnels chapeautés par les nouveaux maitres africains dans les quatre échantillons narratifs. Dans les trois romans et le film, le désordre domestique préfigure, reflète ou amplifie le chaos national. L'auteure met également en parallèle la révolte des filles éclairées - Rama dans Xala, Raabi dans La grève des bàttus et Daba dans Une si longue lettre - non seulement contre les lois et les aventures libidineuses de leurs pères mais encore contre le conservatisme apparent de leurs mères et l'ordre établi ankylosant. Par ailleurs, la gestion féminine louable du foyer en l'absence de l'apport marital, semble justifier l'impératif de faire gouverner les États africains par des femmes, tel qu'énoncé par Calixthe Beyala: «Les hommes ont mis trente ans pour montrer leur incapacité à gérer un continent, trente ans pour conduire ce continent au bord de la faillite $»^{6}$.

7 Le geste de Sembène, comme celui d'Ahmadou Kourouma, d'Emmanuel Dongala, de Koffi Kwahulé et de Gustave Akakpo de prendre fait et cause pour les femmes en intégrant leurs perspectives à l'histoire/Histoire et en les transformant en forces agissantes crédibles, les démarque du Chinua Achebe de Things Fall Apart. Ce constat amène Andrade à faire observer que les hommes avertis aussi bien que les femmes 
lucides peuvent dépeindre les subtilités de la condition féminine et inscrire dans leurs textes l'apport des femmes africaines à la construction nationale et à la (ré)invention constante de la communauté. Malgré leur dette à l'ouvrage de Fanon, les quatre récits narratifs féministes, dans la mesure où ils féminisent et donc complètent les modèles fanoniens, dépassent la portée parfois misogyne de l'essai.

De la même façon que ces quatre œuvres transcendent le cadre conceptuel de leur texte-source, Nervous Conditions de Tsitsi Dangaremgba et Maps de Nuruddin Farah imitent et également contestent le projet du Bildungsroman occidental de tracer l'individuation et le développement psychosocial du protagoniste, souvent masculin, au sein d'une maturation nationale. Les histoires gémellaires de Tambu et de sa cousine Nyasha, dès l'enfance à travers l'adolescence jusqu'au seuil de l'âge adulte, entretiennent une relation discursive et notionnelle avec les thèses fanonienes formulées dans Les damnés de la terre et aussi avec l'introduction de Sartre au même ouvrage, introduction où le philosophe existentialiste aux allures marxistes, utilise l'expression «nervous condition » pour décrire la déstabilisation de l'indigène colonisé. Dans le roman de Dangaremgba, les contextes familiaux intolérables régis par le code patriarcal font pendant au macro-espace colonial placé sous le signe du racisme. L'évolution de deux filles dans un double contexte aliénant, de niveaux sociaux différents mais d'orientation semblable parce que tirant leur inspiration du pater familias néocolonial, Babamukuru, permet à Dangaremgba de livrer des renseignements genrés sur le devenir national tant il est vrai que l'œuvre postcoloniale se situe à l'intersection de la race, du genre, de la classe et de la révolte. Aussi les conditions nerveuses de l'indigène revêtent-elles chez Dangaremgba des dimensions féminines souvent gommées par les discoureurs et artistes du nationalisme africain.

Dans le roman Maps dont le titre évoque les contours physiques, symboliques et inventés de multiples communautés en coprésence et en rivalité, Somalie, Éthiopie et Ogaden, les trajectoires jumelées de Askar et de sa mère de substitution, Misra, se tissent dans une arène de conflits politiques, identitaires et familiaux. Alors que la région d'Ogaden est disputée par la Somalie et l'Éthiopie, les deux protagonistes évoluent dans un véritable contexte de soupçons car toujours tiraillés entre le flou et le concret, l'errance et la stabilité, d'autant que c'est Misra, d'origine éthiopienne, qui a pourtant enseigné à son fils adoptif la langue, le code ancestral et l'esprit communautaire somaliens qu'elle-même avait appris aux autres. Mirsa est configurée par Askar comme absence et présence, étrangère et compatriote, ennemie et femme désirée. Si le fils, devenu adulte, se croit dans ses droits patriotiques de commettre le "matricide» en tuant cette mère sociale qu'il soupçonne d'avoir trahi la cause nationale, il se fait en même temps et de manière ironique, un traitre, dans la mesure où la femme picaresque assassinée fait figure de mère-terre et de mère-patrie. Dans son geste peut se lire aussi une tentative confuse de se libérer de ses fantasmes et encore la peur de l'Autre, celle-là même à qui il doit sa vie. Dès lors, le terme "formation » dans le titre du chapitre tire sa justification du terme Bildung tandis que le mot « deformation » évoque le parcours nébuleux des personnages problématiques que sont Nyasha dans Nervous Conditions et Askar dans Maps.

Dans le quatrième chapitre intitulé «Bildung at the Boundaries », Andrade utilise pour texte d'appui deux romans de l'académicienne Assia Djebar, L'amour, la fantasia et Ombre sultane. Le titre laisse voir le souci de situer l'analyse dans la zone grise du Bildungsroman pour établir en parallèle l'évolution chaotique des sujets féminins au sein 
d'une nation déchirée qu'est l'Algérie. Ainsi le récit familial des femmes se fait-il une allégorie de la reconstruction nationale. Les multiples vignettes qui composent L'amour, la fantasia offrent des incursions dans la participation des femmes à la résistance algérienne lors de l'annexion et des guerres de libération nationale. Moins directe dans Ombre sultane, Djebar fait dialoguer ce roman, élaboré autour d'Isma et Hajila, avec le chef d'œuvre de la littérature arabe, Mille et Une Nuits. Dans l'hypotexte comme dans l'hypertexte, l'homosociabilité féminine conduit les femmes à l'emporter sur la tyrannie familiale étouffante. En dehors de cette relation inter-discursive et généalogique avec un classique s'élabore un réseau d'échanges et de clins d'œil entre une pluralité de destinées féminines tant dans les deux récits de Djebar que dans les autres œuvres du corpus. Comme l'a bien souligné Andrade, Misra, l'étrangère devenue figure nationale énigmatique dans Maps, évoque Pauline Roland, la Française qui prend les allures d'une mère nationale algérienne dans L'amour, la fantasia.

11 Andrade l'a bien démontré: si les œuvres féminines offrent rarement des représentations explicites de combats politiques, leurs peintures diverses de la famille, noyau de la nation, relèvent d'une réinvention subtile de l'État. Ainsi conceptualisés, les tableaux familiaux constituent des mises en abyme de la destinée nationale, les enjeux s'avérant souvent les mêmes pour le microcosme comme pour le macrocosme. Par ailleurs, l'engagement politique des écrivaines rejoint celui de leurs homologues masculins de tendance féministe et/ou d'inclination ouvertement publique.

Dans cette mosaïque brillante sertie de bonnes traductions et d'anglais soutenu mais limpide, l'auteure redynamise le récit nationaliste en recentrant l'apport féminin à la chose politique et à la figuration de la cité. Soucieuse de faire ressortir les différences, les interstices et les passerelles, l'auteure revalorise dans un premier registre la notion de féminisme pluriel, dans un esprit poststructuraliste qui reconnaît les particularités sociohistoriques, et dans un second temps, établit les liens généalogiques entre textes et leurs sources-textes, entre textes et Histoire, entre personnages féminins multiples à l'intérieur des textes et entre divers textes.

\section{NOTES}

1. J. M. NFAH-ABBENY, Gender in African Women's Writing: Identity, Sexuality and Difference, Bloomington, Indiana University Press, 1997.

2. P. HERZBERGER-FOFANA, Littérature féminine francophone d'Afrique noire, suivi d'Un Dictionnaire des romancières, Paris, L'Harmattan, 2000.

3. K. HARROW, Less than One and Double: A Feminist Reading of African Women's Writing, Porthsmouth, Heinemann, 2002.

4. A. A. COLY, The Pull of Postcolonial Nationhood: Gender and Migration, Lanham, Lexington, 2010.

5. L. SENGHOR, La violation d'un pays et autres écrits anticolonialistes, Paris, L'Harmattan, 2012 [1927]. 
6. É. BRIÈRE, « Le retour des mères dévorantes ", Notre Librairie, 117, 1994, p. 69.

7. L'aliénation socioculturelle est également thématisée dans Peau noire, masques blancs (du même auteur). 